\title{
The emerging therapy with probiotics in the management of inflammatory bowel disease: current status
}

\author{
Rajiv Kumar*, Jagjit Singh
}

Department of Pharmacology, Government Medical College \& Hospital, Chandigarh-160030, India

Received: 27 May 2013

Accepted: 14 June 2013

*Correspondence to:

Dr. Rajiv Kumar,

Email: drrajiv.08@gmail.com

(C) 2013 Kumar R et al. This is an open-access article distributed under the terms of the Creative Commons Attribution License, which permits unrestricted use, distribution, and reproduction in any medium, provided the original work is properly cited.

\begin{abstract}
Inflammatory Bowel Disease (IBD) comprises Ulcerative Colitis (UC) and Crohn's Disease (CD) with unknown aetiology. Most of the drugs used to treat IBD as standard treatment produce adverse effects during long term therapy. Evidence has suggested a role of intestinal microbiota in IBD. The use of probiotics and prebiotics is the natural approach to treat IBD. The objective of this article was to review the studies on probiotics that cover the therapeutic status in Inflammatory Bowel Disease. Appraisal of published articles from peer reviewed journals, search from PubMed and Wiley Blackwell website for English language publications using defined key words according to disease type. Studies have shown that probiotic agents play an important role in IBD and these are VSL\#3, Bifido-ferminted milk, Escherichia coli Nissle 1917, Saccharomyces boulardi and "BIO-THREE for inducing remission in patients with active UC, for preventing relapses in inactive UC patients and also in UC patients with ileo-anal pouch anastomosis. Lactobacillllus rhamnosus GG and Lactobacilllus johnsonii LA1 can prevent endoscopic recurrences in patients with inactive CD. Probiotic intervention study designs in IBD patients searched were RCT vs Placebo / RCT vs standard treatment . Studies - with uncontrolled design, - with prebiotics intervention and with helminths were also searched. There is a promising role of probiotics and prebiotics in chronic mucosal inflammation that occurs in Inflammatory Bowel Disease. Sufficient evidence to support the role of probiotics in CD are not available. Well designed RCT studies based on intention -to- treat analyses are required.
\end{abstract}

Keywords: Probiotics, Inflammatory Bowel Disease, Ulcerative Colitis, Crohn's Disease

\section{INTRODUCTION}

Inflammatory Bowel Disease (IBD) - chronic relapsing inflammatory disorder of GIT, Comprises - ulcerative colitis (UC) and crohn's disease (CD) with heterogenic clinical presentation, aetiology is not clear. Ulcerative colitis (UC) involve rectum and extend proximally in to colon and may involve small intestine, UC is characterized by continuous superficial mucosal inflammation limited to colon. Crohn's disease (CD) involve segment of both small and large intestine with transmular involvement leading to stricture and bowel obstruction, and is frequent in ileum and colon, but can affect any portion of gut, associated with intestinal granuloma and complicated by stricture and fistula. ${ }^{1,2}$ Complex interplay of genetic, immunological, microbiological, environmental factor, and psychic factor are known to play a role. An antigen initiate the inflammatory process leading to cascade of proinflammatory events and proinflammatory mediator are cytokines like TNF $-\alpha$ and free radicals. The aim of the treatment is to provide clinical remission and endoscopic remission is the secondary goal. Standard medical therapy includes corticosteroids in the acute phase, mesalazine and immunomodulators to maintain remission and biological agent for refractory and for severe cases. Most of the drugs used to treat IBD as standard treatment produce adverse effects during long term therapy, such as leukopenia, liver function abnormalities because of non specific suppression of immune system can develop opportunistic infections except mesalazine. ${ }^{1-3)}$ Increase risk of lymphoma with thiopurine, TNF - $\alpha$ blocking agents. ${ }^{4,5}$ Body of evidence suggested a role of enteric microbial flora (intestinal microbiota) in inducing and maintaining 
intestinal inflammation with gut immune system in patients with Inflammatory Bowel Disease. ${ }^{6,7}$

A safer therapeutic alternative is offered by probiotics with or without addition of prebiotics a natural approach to IBD treatment. The yeast saccharomyces boulardii with mesalazine has been shown to maintain remission of inactive crohn's disease more effectively than mesalazine alone. ${ }^{8}$ VSL\#3 a probiotic mixture i.e. consisting of four strains of Lactobacillus (L Casei, L Plantarum, L acidophilus and $\mathrm{L}$ delbrueckii subsp. Bulgaricus), three strains of Biofidobacterium (B llongum, B brave and B infants) and one strain of Streptococcus ( $\mathrm{S}$ salivarius subsp. Thermophilus) has been shown to prevent recurrence of pouchitis in several clinical trial, but has provided disappointing results in experimental model. ${ }^{9}$

Hence the observation carried out in experimental models does not necessarily predict same in clinical trials. There is the potential role of probiotics with or without addition of prebiotics in IBD. ${ }^{9,10}$

Studies were reviewed on probiotics that cover the therapeutic status in Inflammatory Bowel Disease. Search Strategies was the appraisal of published articles from peer reviewed journals, search from PubMed and Wiley Blackwell website for English language publications using defined key words according to disease type.

\section{NORMAL INTESTINAL MICROBIOTA}

Sterile GIT at birth rapidly colonized by successive waves of microorganism, comprising. ${ }^{13-14}$ bacteria and stabilizes at the time of weaning. ${ }^{11,12}$ Human intestinal microbial composition present in three clusters or Enterotypes and are Bacteroides, Prevotella, and Ruminococcus. ${ }^{13}$ The denisity and diversity increases from stomach to colon, high interindividual diversity and microbiota is dominated by the phyla Firmicutes and Bacteroidetes. $^{12,14,15}$

Production of short chain fatty acid (SCFAs) i.e. acetate propionate and butyrate by saccharolytic bacterial fermentation of nondigestable carbohydrate. SCFAs-areenergy source for intestinal epithelial cell - affect cell proliferation, differentiation, mucus secretion and barrier function, and provide antiinflammatory and anti-oxidant function. ${ }^{7,13,16}$

\section{Inflammatory Bowel Disease and Changes that occur in intestinal microbiota}

Studies have suggested that intestinal microbiota changes, mucosal bacterial counts are higher in IBD patients. ${ }^{17,18}$ Antibiotic showed a significant benefit over placebo for inducing remission in CD and UC. ${ }^{19}$ Fewer fremicutes was found and low bacterial count for Faecalibacterium Prausnitz $l l^{20-23}$ and bifidobacteria, ${ }^{24,25}$ SCFAs production reduced. Faecal microbiota less diverse. $^{26,27}$ Diversity of bacteroidetes phylum also reduced. ${ }^{15}$ Increase of enterobacteriaceae, and E. coli $^{22,25,28}$, decrease faecal concentration of butyrate in $\mathrm{UC},{ }^{12,29}$ and significant decreases of the transcriptional activity of the mucosa associated microbiota -IBD. ${ }^{21}$ Molecular approaches like FISH, PCR and pyrosequencing have identified a microbial disbiosis in IBD patients. ${ }^{26,27}$ CARDIS $-1^{\text {st }} \mathrm{CD}$ gene identified. ${ }^{30,31}$

\section{Other changes that occur in IBD}

Loss of oral tolerance to commensal bacteria in IBD, increase response to commensal bacteria - contributing the intestinal inflammation. Increase mucosal infiltration of CD4 - lymphocytes, dysfunctional dendrite cells, dysregulated macrophage induced immune response and abnormalities in regulatory pathway have been reported, ${ }^{32,33}$ and in $\mathrm{CD}$ increase production of the per (Th)-1 cytokines and the Th17 cytokine interleukin (IL)17 have been observed. ${ }^{7,33,34}$ In UC, preferential expression of Th- 2 cytokines, IL-4, IL- 5 and increase in IL-17 have been observed. ${ }^{32,33,35}$ In both UC and CD reduced number of regulatory $\mathrm{T}$ cell have been observed. ${ }^{7,32}$

Reduced number of regulatory $\mathrm{T}$ cell, and genetic susceptibility observed in IBD will contribute to the loss of oral tolerance. ${ }^{36}$

\section{PROBIOTICS}

Probiotics are "live microorganisms, which when administered in adequate amount confer a health benefit to the host". ${ }^{37,38}$ At the start of the $20^{\text {th }}$ century, Russian Nobel prize winner Elie Metchnikoff, ${ }^{39}$ a scientist at the Pasteur Institute, was the first to conceptualize "Probiotics", and the term coined in 1965 by Lilly and Stillwell. (40) Criteria: They should be of human origin, must be safe, genetically stable and able to survive passage through the GIT (* low $\mathrm{pH}$, bile and digestive enzymes), different bacterial strain can have different effects, they may act complimentarily or even synergistically. ${ }^{5}$ Probiotics are bacteria, these are- lactic acid bacteria, Lactobacillus acidophilus, L. casei, L. lactis, L. helviticus, L. salivarius, L. plantrum, L. bulgaricus, L. rhamnosus, L. johnsonii, L. reuteri, L. fermentum, L. delbrueckii, Streptococcus thermophilus, Enterococcus faecium, E. faecalis, Bifidobacterium bifidum, B. breve, B. longum and Saccharomyces boulardii are commonly used probiotics. ${ }^{41}$

The probiotics when attached to the wall of intestine increase the number of beneficial bacteria and fight against harmful bacteria thus maintaining a balance between the beneficial and harmful bacteria by following mechanisms: Production of inhibitory substances, Blocking of adhesion sites by competitive inhibition, Competition for nutrients otherwise consumed by pathogenic microorganisms, Stimulation of immunity. ${ }^{45,46}$ 
Prebiotics are indigestible carbohydrates, which stimulate the growth of particular species of the microflora of the host, resulting in an ameliorated enteric function. These nondigestible food constituents act primarily by increasing the population of certain bacteria and thus quantitatively altering the microflora. ${ }^{41}$ When reaching the colon, they are fermented by anaerobic bacteria, producing short-chain fatty acid (SCFA) and gas $\left(\mathrm{CO}_{2}\right.$ and $\left.\mathrm{H}_{2}\right)$. As a result, intraluminal $\mathrm{pH}$ drops, ${ }^{42}$ favouring the increase of Bifidobacteria, Lactobacilli and nonpathogenic E. coli and decreasing Bacterodaceae. These are Lactulose, Germinated barley foodstuff, Fructo-oligosaccharides, and Goat's milk oligosaccharides. $^{41}$

Synbiotics are substances containing both probiotics and prebiotics, ${ }^{41}$ synbiotics introduced as "pharmabiotics" by Shanahan. $^{43}$

\section{PROBIOTIC INTERVENTION STUDIES RETRIEVED IN INFLAMMATORY BOWEL DISEASE WERE:}

A- Probiotic intervention studies retrieved in adult

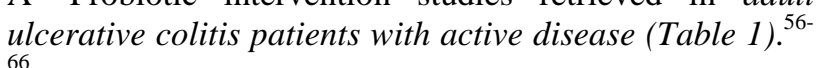

B- Probiotic intervention studies retrieved in adult patients with ulcerative colitis in remission (Table 2). ${ }^{67-73}$

C- Probiotic intervention studies retrieved in adult ulcerative colitis patients with an ileo-anal pouch anastomosis (Table 3). ${ }^{74-77}$

D- Probiotic intervention studies retrieved in adult patients with Crohn's disease (Table 4). ${ }^{78-81}$

Table 1: Probiotic intervention studies retrieved in adult ulcerative colitis patients with active disease.

\begin{tabular}{|c|c|c|c|}
\hline Intervention (daily dose*) & $\begin{array}{l}\text { Disease } \\
\text { activity }\end{array}$ & Design & Clinical outcome \\
\hline $\begin{array}{l}\text { Saccharomyces boulardii }(750 \mathrm{mg})+ \\
\text { mesalazine }(3 \mathrm{~g})\end{array}$ & $\begin{array}{l}\text { Mild to } \\
\text { moderate } \\
\text { active }\end{array}$ & $\begin{array}{l}\text { Uncontrolled } \\
4 \mathrm{wk} \\
(\mathrm{N}=25)\end{array}$ & $\begin{array}{l}68 \% \text { in remission, decreased } \\
\text { clinical activity-significant }\end{array}$ \\
\hline $\begin{array}{l}\text { Bifido-fermented milk } \\
\text { [Bifidobacterium breve, } \\
\text { Bifidobacterium bifidum and } \\
\text { Lactobacillus acidophilus] }\left(10 \times 10^{9}\right) \\
\text { Vs PL }\end{array}$ & $\begin{array}{l}\text { Mild to } \\
\text { moderately } \\
\text { active }\end{array}$ & $\begin{array}{l}\mathrm{RCT} \text { Vs no } \\
\text { additive tx, } \\
12 \mathrm{mo}(\mathrm{N}=21)\end{array}$ & $\begin{array}{l}\text { Significant relapse rate, } \& \text { no } \\
\text { differences in colonoscopic } \\
\text { findings } 57,58\end{array}$ \\
\hline $\begin{array}{l}\text { VSL \# } 3\left(9 \times 10^{11}\right)+\text { balsalazide } \\
(2.25 \mathrm{~g}) \text { Vs balsalazide }(4.5 \mathrm{~g}) \text { Vs } \\
\text { Mesalazine }(2.4 \mathrm{~g})\end{array}$ & Moderately active & $\begin{array}{l}\text { RCT Vs standard } \\
\text { tx, 8wk }(\mathrm{N}=90)\end{array}$ & $\begin{array}{l}\text { Significant remission rate, \& } \\
\text { faster remission induction }\end{array}$ \\
\hline VSL \# $3\left(3.6 \times 10^{12}\right)$ & Moderately active & $\begin{array}{l}\text { Uncontrolled, 6wk } \\
(\mathrm{N}=34)\end{array}$ & $\begin{array}{l}53 \% \text { entered remission, } 77 \% \\
\text { decreased }>3 \text { points in clinical } \\
\text { activity index }\end{array}$ \\
\hline $\begin{array}{l}\text { BIO-THREE (Streptococcus faecalis } \\
18 \mathrm{mg} \text {, Clostridium butyricum } 90 \mathrm{mg} \text {. } \\
\text { Bacillus mesentericus } 90 \mathrm{mg} \text { ) [ } \mathrm{n}=10 \text {; } \\
\text { also } 100 \mathrm{~g} \text { dietary fibre daily] }\end{array}$ & $\begin{array}{l}\text { Mild to } \\
\text { moderately } \\
\text { refractory active }\end{array}$ & $\begin{array}{l}\text { Uncontrolled, 4wk } \\
(\mathrm{N}=20)\end{array}$ & $45 \%$ in remission. ${ }^{61}$ \\
\hline VSL\#3 $\left(1.8 \times 10^{12}\right)$ & $\begin{array}{l}\text { UC pts } \\
\text { (active+inactive) }\end{array}$ & $\begin{array}{l}\text { Uncontrolled, 5wk } \\
(\mathrm{N}=15)\end{array}$ & $\begin{array}{l}\text { Decrease in clinical disease } \\
\text { activity. }{ }^{2}\end{array}$ \\
\hline $\begin{array}{l}\text { Bifidobacterium longum Bb536 } \\
\left(2-3 \times 10^{11}\right)\end{array}$ & Active & $\begin{array}{l}\text { Uncontrolled, } \\
24 w k \\
(\mathrm{~N}=14)\end{array}$ & $67 \%$ reached remission. ${ }^{63}$ \\
\hline VSL\#3 $\left(3.6 \times 10^{12}\right)$ vs PL & $\begin{array}{l}\text { Mild to } \\
\text { moderately active }\end{array}$ & $\begin{array}{l}\text { RCT vs PL, } 12 \mathrm{wk} \\
(\mathrm{N}=147)\end{array}$ & $\begin{array}{l}>50 \% \text { improved disease activity } \\
\text { at wk } 6 \text {, remission at wk } 12 \text { - } \\
\text { significant }{ }^{64}\end{array}$ \\
\hline $\begin{array}{l}\text { Escherichia coli Nissle } 1917 \text { enema } \\
\left(4 \times 10^{9}\right) \text { Vs }\left(2 \times 10^{9}\right) \text { Vs }\left(10^{9}\right) \text { Vs PL. }\end{array}$ & $\begin{array}{l}\text { Mild to } \\
\text { moderately active }\end{array}$ & $\begin{array}{l}\text { RCT Vs PL, 8wk } \\
(\mathrm{N}=90)\end{array}$ & $\begin{array}{l}\text { Significant remission rates as } \\
\text { per analysis }{ }^{65}\end{array}$ \\
\hline $\begin{array}{l}\text { 5-ASA }(2.4 \mathrm{~g}) \text { Vs 5-ASA + } \\
\text { Lactobacillus casei }\left(1.6 \times 10^{9}\right) \text { orally } \\
\text { Vs 5-ASA + L. casei }\left(1.6 \times 10^{9}\right) \\
\text { rectally. }\end{array}$ & Mild active & $\begin{array}{l}\text { RCT Vs standard } \\
\text { tx, 8wk }(\mathrm{N}=26)\end{array}$ & $\begin{array}{l}\text { Improved clinical activity in 5- } \\
\text { ASA group. Improved } \\
\text { histology in both L. casei } \\
\text { groups. }\end{array}$ \\
\hline
\end{tabular}

Daily dose* in CFU=colony-forming units; pts=patients; $\mathrm{PL}=$ placebo; $\mathrm{tx}=$ treatment; $\mathrm{mo}=\mathrm{months}$; $\mathrm{N}=$ number of patients; $\mathrm{RCT}=$ randomized controlled trial; $\mathrm{UC}=$ ulcerative colitis; 5-ASA=5- aminosalicylic acid 
Table 2: Probiotic intervention studies retrieved in adult patients with ulcerative colitis in remission.

\begin{tabular}{|c|c|c|c|}
\hline Intervention (daily dose*) & $\begin{array}{l}\text { Disease } \\
\text { activity }\end{array}$ & Design & Clinical outcome \\
\hline $\begin{array}{l}\text { Escherichia coli Nissle } 1917 \\
\text { enema }\left(50 \times 10^{9}\right) \text { Vs mesalazine } \\
(3 \times 500 \mathrm{mg})\end{array}$ & Inactive & $\begin{array}{l}\text { RCT Vs standard } \\
\text { tx, } 12 \mathrm{wk}(\mathrm{N}=103)\end{array}$ & $\begin{array}{l}\text { Similar relapse rate, } \\
\text { NS. }\end{array}$ \\
\hline $\begin{array}{l}\text { E.Coli Nissle } 1917\left(50 \times 10^{9}\right) \mathrm{Vs} \\
\text { mesalazine }(3 \times 400 \mathrm{mg})\end{array}$ & $\begin{array}{l}\text { Inactive (after } \\
\text { remission } \\
\text { induction) }\end{array}$ & $\begin{array}{l}\text { RCT Vs standard } \\
\text { tx, } 12 \mathrm{mo}(\mathrm{N}=83)\end{array}$ & $\begin{array}{l}\text { Similar relapse rate, } \\
\text { NS. }\end{array}$ \\
\hline VSL \# $3\left(3 \times 10^{12}\right)$ & $\begin{array}{l}\text { Inactive } \\
\text { (intolerant / } \\
\text { allergic to 5- } \\
\text { ASA) }\end{array}$ & $\begin{array}{l}\text { Uncontrolled } 12 \mathrm{mo} \\
(\mathrm{N}=20)\end{array}$ & $\begin{array}{l}75 \% \text { maintained } \\
\text { remission. } 69\end{array}$ \\
\hline $\begin{array}{l}\text { Bifid triple viable capsule }(1.26 \mathrm{~g}) \\
\text { vs PL }\end{array}$ & $\begin{array}{l}\text { Inactive after } \\
\text { inducing } \\
\text { remission }\end{array}$ & $\begin{array}{l}\text { RCT vs PL, } 8 w k \\
(\mathrm{~N}=30)\end{array}$ & Significant relapse rate..$^{70}$ \\
\hline $\begin{array}{l}\text { Lactobacillus rhamnosus GG } \\
\left(18 \times 10^{9}\right) \text { vs mesalazine }(2400 \mathrm{mg}) \\
\text { vs L.GG }\left(18 \times 10^{9}\right)+\text { mesalazine } \\
(2400 \mathrm{mg})\end{array}$ & Inactive & $\begin{array}{l}\text { RCT vs standard tx, } \\
12 \mathrm{mo}(\mathrm{N}=187)\end{array}$ & $\begin{array}{l}\text { Similar relapse rate, NS. } \\
\text { No difference in clinical, } \\
\text { endoscopic and } \\
\text { histological scores. }\end{array}$ \\
\hline $\begin{array}{l}\text { Saccharomyces boulardii } \\
(500 \mathrm{mg})+\text { rifaximin }(400 \mathrm{mg})\end{array}$ & $\begin{array}{l}\text { Inactive } \\
\text { (mesalamine } \\
\text { intolerant) }\end{array}$ & $\begin{array}{l}\text { Uncontrolled, 3mo } \\
(\mathrm{N}=6)\end{array}$ & $\begin{array}{l}\text { Maintained remission } \\
\text { based on clinical } \\
\text { activity. }\end{array}$ \\
\hline $\begin{array}{l}\text { Lactobacillus acidophilus } \\
\text { (La-5) + Bifidobacterium } \\
\text { animalis lactis [Bb-12] } \\
\left.91.5 \times 10^{11}\right) \text { vs Pl }\end{array}$ & Inactive & $\begin{array}{l}\text { RCT vs PL, 52wk } \\
(\mathrm{N}=32)\end{array}$ & Maintenance remission. ${ }^{73}$ \\
\hline
\end{tabular}

Daily dose* in $\mathrm{CFU}=$ colony-forming units; $\mathrm{PL}=$ placebo; tx=treatment; mo=months; $\mathrm{N}=$ number of patients; NS=not significant; RCT=randomized controlled trial; 5-ASA=5- aminosalicylic acid.

Table 3: Probiotic intervention studies retrieved in adult ulcerative colitis patients with an ileo-anal pouch anastomosis.

\begin{tabular}{|c|c|c|c|}
\hline Intervention (daily dose*) & Disease activity & Design & Clinical outcome \\
\hline $\begin{array}{l}\text { Escherichia coli Nissle } 1917 \\
\left(2.5-5 \times 10^{10}\right)\end{array}$ & Active pouchitis & $\begin{array}{l}\text { Uncontrolled, } \\
315 / 56 \mathrm{~d}(\mathrm{~N}=2)\end{array}$ & $\begin{array}{l}\text { Both in remission from day } \\
50 \text { and } 5 \text {, respectively. }\end{array}$ \\
\hline VSL \# $3\left(36 \times 10^{11}\right)$ & $\begin{array}{l}\text { Mild to active } \\
\text { pouchitis }\end{array}$ & $\begin{array}{l}\text { Uncontrolled 4wk } \\
(\mathrm{N}=23)\end{array}$ & $\begin{array}{l}69 \% \text { in remission, Decreased } \\
\text { PDAI. }^{75}\end{array}$ \\
\hline VSL \# $3\left(18 \times 10^{11}\right)$ vs PL & $\begin{array}{l}\text { After induction } \\
\text { remission by } \\
\text { antibiotics }\end{array}$ & $\begin{array}{l}\text { RCT vs PL, 9mo } \\
(\mathrm{N}=40)\end{array}$ & Significant relapse rate. ${ }^{76}$ \\
\hline $\begin{array}{l}\text { Lactobacillus rhamnosus } \\
\text { GG }\left(2-4 \times 10^{10}\right) \text { vs PL }\end{array}$ & $\begin{array}{l}\text { With history of } \\
\text { pouchitis (subgroup } \\
\text { had pouchitis) }\end{array}$ & $\begin{array}{l}\text { RCT vs PL, } 3 \mathrm{mo} \\
(\mathrm{N}=20)\end{array}$ & $\begin{array}{l}\text { No change in PDAI scores } \\
\text { between groups. }^{77}\end{array}$ \\
\hline
\end{tabular}

Daily dose* in CFU=colony-forming units; $\mathrm{PL}=$ placebo; mo=months; $\mathrm{N}=$ number of patients; RCT=randomized controlled trial; PDAI=Pouch Disease Activity Index. 
Table 4: Probiotic intervention studies retrieved in adult patients with Crohn's disease.

\begin{tabular}{|c|c|c|c|}
\hline Intervention (daily dose*) & Disease activity & Design & Clinical outcome \\
\hline $\begin{array}{l}\text { Prednislon }+ \text { Escherichia coli } \\
\text { Nissle } 1917\left(5 \times 10^{10}\right) \text { vs } \\
\text { prednisolon }+ \text { PL }\end{array}$ & Active, all colon & $\begin{array}{l}\text { RCT vs PL, } 12 \mathrm{mo} \\
(\mathrm{N}=23)\end{array}$ & $\begin{array}{l}\text { Entered in remission, \& } \\
\text { relapse rate NS. }{ }^{78}\end{array}$ \\
\hline $\begin{array}{l}\text { Lactobacillus acidophilus, } \\
\text { Bifidobacterium, } \\
\text { Lactobacillus casei }+ \\
\text { Streptococcus salivarius } \\
\text { subsp. Thermophilus }\left(8 \times 10^{9}\right)\end{array}$ & $\begin{array}{l}\text { Active, disease } \\
\text { locations unknown }\end{array}$ & $\begin{array}{l}\text { Uncontrolled (case } \\
\text { reports), 7-12mo } \\
(\mathrm{N}=3)\end{array}$ & $\begin{array}{l}\text { Maintained remission but } \\
1 \text { wk abdominal pain } \\
\text { (after } 7 \text { and } 8 \mathrm{mo} \text { ) in } 2 \text { of } \\
3 \text { pts. }^{79}\end{array}$ \\
\hline $\begin{array}{l}\text { Saccharomyces boulardii } \\
(1 g)+\text { mesalazine }(2 g) \text { vs } \\
\text { mesalazine }(3 g)\end{array}$ & $\begin{array}{l}\text { 33-Inactive, }(9 \text { ileum, } 1 \\
\text { colon, } 23 \text { ileum }+ \\
\text { colon })\end{array}$ & $\begin{array}{l}\text { RCT vs standard therapy, } \\
6 \mathrm{mo}(\mathrm{N}=33)\end{array}$ & Significant relapse rate. ${ }^{80}$ \\
\hline $\begin{array}{l}\text { Lactobacillus rhamnosus GG } \\
\left(12 \times 10^{9}\right) \text { vs PL }\end{array}$ & $\begin{array}{l}45 \text {-Inactive }(10 \mathrm{~d} \text { after } \\
\text { curative resection, } \\
35 \text { ileum, } 3 \text { colon, } 7 \\
\text { ileum + colon) }\end{array}$ & $\begin{array}{l}\text { RCT vs PL, } 12 \mathrm{mo} \\
(\mathrm{N}=45)\end{array}$ & $\begin{array}{l}\text { Clinical relapse \& had } \\
\text { endoscopic recurrence of } \\
\text { those in remission. }{ }^{81}\end{array}$ \\
\hline
\end{tabular}

Daily dose* in CFU=colony-forming units; PL=placebo; mo=months; $\mathrm{N}=$ number of patients; RCT=randomized controlled trial;

\section{Role of Helminths in Inflammatory Bowel Disease:}

There is evidence from studies that Inflammatory Bowel Disease (IBD) is much less common in countries with poor sanitation and low hygiene levels, where helminth infections are common, in comparison with Western countries. ${ }^{54}$ It has thus been assumed that helminths may lead to the prevention of IBD by some unknown mechanism. Studies have revealed, the use of helminths such as Trichuris suis for the treatment of IBD patients, ${ }^{55}$ and helminths are in clinical trial.

\section{DISCUSSION AND CONCLUSIONS}

Studies have revealed that probiotics affect the composition of the microbial ecosystem by competition of nutrients and adhesion sites, by the production of antimicrobial substances and / or via cell-cell communication. ${ }^{44,45}$ Probiotics affect host immune system by interaction of bacterial products, cell wall components or DNA with epithelial and gut-associated immune cell. ${ }^{46}$ There is evidence from studies that probiotics causes changes in cytokine production, modulation in dendritic cell function, and increase of natural killer cell activity, and induction of regulatory $\mathrm{T}$ cell and defensins. ${ }^{44,46,47}$

Probiotics contribute to SCFAs, butyrate / or affect barrier function by induction of mucin secretion, by enhancement of tight junction expression and functioning, ${ }^{48,49}$ also probiotics decreases epithelial cell apoptosis.
There is evidence from studies that intestinal microbiota play a role not only in the chronic mucosal inflammation in IBD but also in Irritable Bowel Syndromes (IBS), Obesity, and the Metabolic Syndrome., ${ }^{2,50-53}$

The main rationale for probiotics interventional studies is the manipulation of the indigenous intestinal microbiota composition and activity, the immune system and host barrier function. ${ }^{44,46,47}$ Studies have shown that probiotic agents play an important role in IBD, These are VSL\#3, Bifido- ferminted milk, Escherichia coli Nissle 1917, Saccharomyces boulardii and "BIO-THREE for inducing remission in patients with active $\mathrm{UC}$, for preventing relapses in inactive UC patients and also in UC patients with ileo-anal pouch anastomosis. Lactobacillllus rhamnosus GG and Lactobacillllus johnsonii LA1 can prevent endos -copic recurrences in patients with inactive CD. Probiotic intervention study designs in IBD patients searched were RCT vs Placebo / RCT vs standard treatment.

Studies with uncontrolled design, with prebiotics intervention and with helminths were also searched. There is a promising role of probiotics and prebiotics in chronic mucosal inflammation that occurs in Inflammatory Bowel Disease. Sufficient evidence to support the role of probiotics in $\mathrm{CD}$ are not available. Well designed RCT studies based on intention -to- treat analyses are required.

Funding: No funding sources Conflict of interest: None declared

Ethical approval: Not required 


\section{REFERENCES}

1. Bengmark S. Bioecological control of inflammatory bowel disease. Clin Nutr 2007;26:169-81.

2. Talley NJ, Abreu MT, Achkar JP et al. An evidencebased systematic review on medical therapies for inflammatory bowel disease. Am J Gastroenterol 2011 Apr;106 Suppl.1:S2-25;Quiz 56.

3. Dignass A, Van Assche, Lindsay JO et al. The second European evidence-based consensus on the diagnosis and management of Crohn's disease: current management. J Crohn's Colitis 2010 Feb;4(1):28-62.

4. Herrinton LJ, Weng X, Liu L et al. Role of thiopurine and anti-TNF therapy in lymphoma in inflammatory bowel disease. Am J Gastroentrol 2011 Dec;106(12):2146-53.

5. Timmerman HM, Koning CJ, Mulder L et al. Monostrain, multistrain and multispecies probiotics: a comparison of functionality and efficacy. Int Food Microbiol 2004 Nov 15;96 (3):219-33.

6. Guslandi M.Antibiotics for inflammatory bowel disease: do they work? Eur J Gastroenterol Hepatol 2005; 17: 145-7.

7. Thompson-Chagoyan OC, Maldonado J, Gill A. Aetiology of inflammatory bowel disease (IBD): role of intestinal microbiota and gut-associated lymphoid tissue immune response. Clin Nutr 2005 Jun; 24 (3):339-52.

8. Guslandi $M$, et al. Saccharomyces boulardii in maintenance treatment of Crohn's disease. Dig Dis Sci 2000 Jul ; 45 (7): 1462-4.

9. Damaskos D, Kolios G. Probiotics and prebiotics in inflammatory bowel disease: microflora 'on the scope'. Br J Clin Pharmacol 2008; 65:453-67.

10. Asakura H, Suzuki K, Honma T. Recent advances in basic and clinical aspects of inflammatory bowel disease; which steps in the mucosal inflammation should we block for the treatment of inflammatory bowel disease? World J Gastroenterol 2007;13:2145-9.

11. Zoetendal EG, Akkermans ADL, Akkermans-van Vliet WM et al. The host genotype affects the bacterial community in the human gastrointestinal tract. Microb Ecol Health Dis 2001;13:129-34.

12. Takaishi $H$, Matsuki $T$ et al. Imbalance in intestinal microflora constitution could be involved in the pathogenesis of inflammatory bowel disease. Int $\mathbf{J}$ Med Microbiol 2008 Jul;298 (5-6):463-72.

13. Arumugam M, Raes J, Pelletier E et al. Enterotypes of the human gut microbiome. Nature 2001 May 12;473 (7346):174-80.

14. Eckburg PB, Bik EM, Bernstein CN et al. Diversity of the human intestinal microbial flora. Science 2005 Jun 10;308(5728):1635-8.

15. Frank DN, St Amand AL, Feldman RA et al. Molecular-phylogenetic characterization of microbial community imbalances in human inflammatory bowel disease. Proc Natl Acad Sci USA 2007 Aug 21; 104(34): 13780-5.
16. Hamer HM, Jonkers D, Venema K et al. Review article: the role of butyrate on colonic function. Aliment Pharmacol Ther 2008 Jan 15;27(2):104-19.

17. Swidsinski A, Ladhoff A, Pernthaler A et al. Muscosal flora in inflammatory bowel disease. Gastroenterology 2002;122(1):44-54.

18. Schultsz C, Ven Den Berg, Ten Kate FW et al. The intestinal mucus layer from patients with inflammatory bowel disease harbors high numbers of bacterial compared with controls. Gastroenterology 1999;117(5):1089-97.

19. Khan KJ, Ullman TA, Ford AC et al. antibiotic therapy in inflammatory bowel disease: a systematic review and meta-analysis. Am J Gastroentrol 2011 Apr;106(4):661-73.

20. Manichanh C, Riggotier-Gois, Bonnaud E et al. Reduced diversity of faecal microbiota in Crohn's disease revealed by a metagenomic approach. Gut 2006 Feb;55 (2):205-11.

21. Rehman A, Lepage P, Nolte A et al. Transcriptional activity of the dominant gut mucosal microbiota in chronic inflammatory bowel disease patients. J Med Microbiol 2010 Sep;59 (Pt 9):1114-22.

22. Walker AW, Sanderson JD, Churcher C et al. High throughput clone library analysis of the mucosaassociated microbiota reveals dysbiosis and differences between inflamed and non-inflamed regions of the intestine in inflammatory bowel disease. BMC Microbiol 2011 Jan 10;11:7.

23. Sokol H, Seksik P, Furet JP et al. Low counts of Faecali-bacterium prausnitzii in colitis microbiota. Inflamm Bowel Dis 2009 Aug;15(8):1183-9.

24. Kleessen B, Kroesen AJ, Bhur J et al. Mucosal and invading bacteria in patients with inflammatory bowel disease compared with controls. Scand J Gastroentrol 2002 Sep;37(9):1034-41.

25. Mylonaki M, Rayment NB, Rampton DS et al. Molecular characterization of rectal mucosaassociated bacterial flora in inflammatory bowel disease. Inflamm Bowl Dis 2005 May;11(5):481-7.

26. Dicksved J, Halfvarson J, Rosenquist $M$ et al. Molecular analysis of the gut microbiota of indentical twins with Crohn's disease. Isme J 2008 Jul;2(7):716-27.

27. Martinez C, Antolin M, Santos J et al. Unstable composition of the fecal microbiota in ulcerative colitis during clinical remission. Am J Gastroenterol 2008 Mar;103(3):643-8.

28. Thomazini CM, Rodrigues MA, Rodrigues MA et al. High prevalence of aggregative adherent Escherichia coli strains in the mucosa-associated microbiota of patients with inflammatory bowel disease. Int $\mathrm{J}$ Med Microbiol 2011 Aug;301(6):475-9.

29. Verina P, Hauck W, Hauck W et al. Organic anions and the diagghea of inflammatory bowel disease. Dig Dis Sci 1988 Nov;33(11):1353-8.

30. Hugot JP, Chamaillard M, Zouali $H$ et al. Association of NOD2 leucine-rich repeat variants with susceptibility to Crohn's disease. Nature 2001 May 31;411 (6837):599-603. 
31. Cho JH, Brant SR. Recent insights into the genetics of inflammatory bowel disease. Gastroentrology 2011 May;140(6):1704-12.

32. Abraham C, Medzhitov R. Interactions between the host innate immune system and microbes in inflammatory bowel disease. Gastroenterology 2011 May;140(6):1729-37.

33. Danese S. Immune and nonimmune components orchestrate the pathogenesis of inflammatory bowel disease. Am J Physiol Gastrointest. Liver Physiol 2011 May:300(5):G716-22.

34. Abraham C, Cho JH. IL-23 and autoimmunity: new insights into the pathogenesis of inflammatory bowel disease. Annu Rev Med 2009;60:97-110.

35. Korzenik JR, Podolsky DK. Evolving knowledge and therapy of inflammatory bowel disease. Nat Rev Drug Discov 2006 Mar;5(3):197-209.

36. Less CW, Parkes $M$, Parkes $M$ et al. New IBD genetics: common pathways with other diseases. Gut 2011 Feb 7;60(12):1739-53.

37. Food and Agriculture Organization of the United Nations, WHO. Joint FAO/WHO expert consultation on evaluation of health nutritional properties of probiotics in food including powder milk with live lactic acid bacteria. Cordoba:2001 Oct(online).

38. Joint Food and Agriculture Organization of the United Nations/World Health Organization Working Group report on drafting guidelines for the evaluation of probiotics in food, London, Ontario, Canada, April 30 and May, 2002.

39. Metchnikoff E. The prolongation of life. Optimistic studies. London: Butterworth-Heinemann, 1907.

40. Lilly DM, Stillwell RH. Probiotics: Growth promoting substances produced by microorganisms. Science 1965; 147:747-8.

41. Bengmark S. Pre-, Pro- and synbiotics. Curr Opin Clin Nutr Metab Care 2001;4: 571-9.

42. Govers MJ et al. Wheat bran affects the site of fermentation of resistant starch and luminal indexes related to colon cancer risk: a study in pigs. GUT; 45: 840-7.

43. Shanahan F. Physiological basis for noval drug therapies used to treat the inflammatory bowel diseases. Pathophysiological basis and prospects for probiotic therapy in inflammatory bowel diseases. Am J Physiol Gstrointest Physiol 2005; 288: G417-21.

44. Lebeer S, Vanderleyden J, De Keersmaecker SC et al. Genes and molecules of lactobacilli supporting probiotic action. Microbial Mol Biol Rev 2008 Dec;72(4):728-64.

45. Sherman PM, Ossa JC, Johnson Henry K. Unraveling mechanisms of action of probiotics. Nutr Clin Pract 2009 Feb-Mar;24(1):10-4.

46. Oelschlaeger TA. Mechanisms of probiotic actions: a review. Int J Med Microbiol 2010 Jan;300(1): 57-62.

47. Karczewski J, Konings I, Troost FJ et al. Regulation of human epithelial tight junction proteins by Lactobacillus plantarum in vivo and protective effects on the epithelial barrier. Am J Physiol Gastrointest Liver Physiol 2010 Jun;298(6):G851-9.

48. Caballero-Franco C, De Simone C, Keller K et al. The VSL\#3 probiotic formula induces mucin gene expression and secretion in colonic epithelial cells. Am J Physiol Gastrointest Livel Physiol 2007 Jan;292(1):G315-22.

49. Ukena SN, Singh A, Dringenberg U et al. Probiotic Escherichia coli Nissle 1917 inhibits leaky gut by enhancing mucosal integrity. PLoS One 2007;2(12):el308.

50. Quigley EM. Gut microbiota and the role of probiotics in therapy. Curr Opin Pharmacol 2011 Oct 11;11(6):593-603.

51. Tilg H. Obesity, metabolic syndrome, and microbiota: multiple interactions. J Clin Gastroenterol 2010 Spe;44 Suppl. 1:S16-8.

52. Thia KT et al. An update on the epidemiology of inflammatory bowel disease in Asia. Am J Gastroenterol 2008 Dec;103(12):3167-82.

53. Kawada M, Arihiro A, Mizoguchi E. Insights from advances in research of chemically induced experimental modles of human inflammatory bowel disease. World J Gastroenterol 2007 Nov 14;13(42): 5581-93.

54. Fiasse R, Latinne D. Intestinal helmonths: a clue explaining the low incidence of inflammatory bowel diseases in Subsaharan Africa? Potential benefits and hazards of helminth therapy. Acta Gastroenterol Belg 2006;69:418-22.

55. Reddy A, Fried B. The use of Trichuris suis and other helminth therapies to treat Crohn's disease. Parasitol Res 2007;100:921-7.

56. Guslandi M, Giollo P, Testoni PA. A pilot trial of Saccharomyces boulardii in ulcerative colitis. Eur $\mathbf{J}$ Gastroenterol Hepatol 2003 Jun; 15(6):697-8.

57. Ishikawa H, Akedo I, Umesaki Y et al. Randomized controlled trial of the effect of bifidobacteria fermented milk on ulcerative colitis. J Am Coll Nutr 2003 Feb;22 (1):56-63.

58. Kato K, Mizuno S, Umesaki Y et al. Randomized placebo-controlled trial assessing the effect of bifidobacteria-fermented milk on active ulcerative colitis. Aliment Pharmacol Ther 2004 Nov 15;20(10):1133-41.

59. Tursi A, Brandimarte G, Giorgetti GM et al. Lowdole balsalazide plus a high-potency probiotic preparation is more effective than balsalazide alone or mesalazine in the treatment of acute mildto-moderate ulcerative colitis. Med Sci Monti 2004 Nov;10(11):PI126-31.

60. Bibiloni R, Fedorak RN, Tannock GW et al. VSL\#3 probiotic-mixture induces remission in patients with active ulcerative colitis. Am J Gastroenterol 2005 Jul;100 (7):1539-46.

61. Tsuda Y, Yoshimatsu Y, Aoki H et al. Clinical effectiveness of probiotics therapy (BIO-THREE) in patients with ulcerative colitis refractory to conventional therapy. Scand J Gastroenterol 2007 Nov;42(11):1306-11. 
62. Soo I, Madsen KL, Tejpar Q et al. VSL\#3 probiotic upregulates intestinal mucosal alkaline sphingomyelinase and reduces inflammation. Can $\mathrm{J}$ Gastroenterol 2008 Mar;22(3):237-42.

63. Takeda Y, Nakase H, Namba K et al. Upregulation of T-bet and tight junction molecules by Bifidobactrium longum improves colonic inflammation of ulcerative colitis. Inflamm Bowel Dis 2009 Nov;15(11):1617-8.

64. Sood A, Midha V, Makharia GK et al. The probiotic preparation, VSL\#3 induces remission in patients with mild-to-moderately active ulcerative colitis. Clin Gastroenterol Hepatol 2009 Nov;7(11):12029el.

65. Tursi A, Brandimarte G, Papa A et al. Treatment of relapsing mild-to-moderate ulcerative colitis with the probiotic VSL\#3 as adjunctive to a standard pharmaceutical treatment: a double-blind, randomized, placebo-controlled study. Am J Gastroenterol 2010 Oct;105 (10) :2218-27.

66. D'Inca $R$, Barollo $M$, Scarpa $M$ et al. Rectal administration of Lactobacillus casei DG modifies flora composition and Toll-like receptor expression in colonic mucosa of patients with mild ulcerative colitis. Dig Dis Sci 2011 Apr;56(4):1178-87.

67. Kruis W, Schutz E, Fric $P$ et al. Double-blind comparison of an oral Escherichia coli preparation and mesalazine in maintaining remission of ulcerative colitis. Aliment Pharmacol Ther 1997 Oct;11(5):853-8.

68. Rembacken BJ, Snelling AM, Hawkey PM et al. Nonpathogenic Escherichia coli versus mesalazine for the treatment of ulcerative colitis: a randomized trial. Lancet 1999;354(9179):635-9.

69. Venturi A, Gionchetti P, Rizzello F et al. Impact on the composition of the faecal flora by a new probiotic preparation: preliminary data on maintenance treatment of patients with ulcerative colitis. Aliment Pharmacol Ther 1999;13(8):1103-8.

70. Cui HH, Chen CL, Wang JD et al. Effects of probiotic on intestinal mucosa of patients with ulcerative colitis. World J Gastroenterol 2004 May $15 ; 10(10): 1521-5$

71. Zocco MA, dal Verme LZ, Cremonini $F$ et al. Efficacy of lactobacillus GG in maintaining remission of ulcerative colitis. Aliment Pharmacol Ther 2006 Jun;23(11):1567-74.

72. Guslandi M. Saccharomyces boulardii plus rifaximin in mesalamine-intolerant ulcerative colitis. J Clin Gastroenterol 2010 May-Jun;44(5):385.

73. Wildt S, Nordgaard I, Hansen U et al. A randomized double-blind placebo-controlled trial with Lactobacillus acidophilus La-5 and Bifidobacterium animals subsp. lactis BB-12 for maintainance of remission in ulcerative colitis. J Crohns Colitis 2011 Apr;5(2):115-21.

74. Kuzela L, Kascak M, Vavrecka A. Induction and maintenance of remission with nonpathogenic Escherichia coli in patients with pouchitis. Am J Gastroenterol 2001 Nov; 96(11):3218-9.

75. Gionchetti P, et al. High- dose probiotics for the treatment of active pouchitis. Dis Colon Rectum 2007 Dec;50 (12):2075-82 ; discussion 82-4.

76. Gionchetti P, et al. Oral bacteriotherapy as maintenance treatment in patients with chronic pouchitis: a double blind placebo-controlled trial. Gastroenterology 2000 Aug; 119 (2):305-9.

77. Kuisma J, et al. Effects of Lactobacillus rhamnosus GG on ileal pouch inflammation and microbial flora . Aliment Pharmacol Ther 2003 Feb 15; 17 (4): 50915.

78. Malchow HA. Crohn's disease and Escherichia coli: a new approach in therapy to maintain remission of colonic Crohn's disease? J Clin Gastroenterol 1997;25(4):653-8.

79. Doman DB, et al. "Ecologic niche" therapy for Crohn's disease with adjunctive rifaximin antibiotic treatment followed by Flora-Q probiotic maintenance therapy. Am J Gastroenterol 2008 Jan;103(1)251-2.

80. Guslandi $M$, et al. Saccharomyces boulardii in maintenance treatment of Crohn's disease. Dig Dis Sci 2000 Jul;45(7):1462-4.

81. Prantera $\mathrm{C}$, et al. Ineffectiveness of probiotics in preventing recurrence after curative resection for :Crohn's disease: a randomised controlled trial with Lactobacillus GG. Gut 2002;51(3):405-9.

doi:10.5455/2319-2003.ijbcp20130803

Cite this article as: Kumar R, Singh J. The emerging therapy with probiotics in the management of inflammatory bowel disease: current status. Int J Basic Clin Pharmacol 2013;2:360-7. 